\title{
Arbitrage and Investment Opportunities
}

\author{
Elyès Jouini* Clotilde Napp ${ }^{\dagger}$
}

September 99

\begin{abstract}
We consider a model in which all investment opportunities are described in terms of cash flows. We don't assume that there is a numéraire, the time horizon is not supposed to be finite, the investment opportunities are not specifically related to the buying and selling of securities on a financial market. In this quite general framework, we show that the assumption of no-arbitrage is essentially equivalent to the existence of a "discount process" under which the "net present value" of any investment is nonpositive. Since most market imperfections, such as short sale constraints, convex cone constraints, proportional transaction costs, no borrowing or different borrowing and lending rates, etc., can fit in our model for a specific set of investments, we then obtain a characterization of the noarbitrage condition in these imperfect models, from which it is easy to derive pricing formulae for contingent claims.

Compared with existing results, our approach allows to consider markets with no numéraire or with a numéraire that is subject to constraints. Besides, we introduce a notion of no-free lunch which is less restrictive than the usual one. Last, we characterize the assumption of no-arbitrage (or no-free lunch) for more general investment opportunities, which enables us to consider investments that are not necessarily related to a market model and, more interestingly, to generalize the results obtained for imperfect markets and to obtain them all in a unified way.
\end{abstract}

Keywords : arbitrage - investment opportunities - numéraire - market imperfections - transaction costs - Yan's theorem

\section{Introduction}

A classical problem in mathematical finance is the pricing of financial assets. This problem is usually solved through the use of the so-called Fundamental Theorem of Asset Pricing (see Harrison and Kreps (1979) or Harrison and Pliska (1981)). This theorem asserts that in a perfect financial model, the assumption of no-arbitrage (which amounts to saying that there is no plan yielding some profit without a countervailing threat of loss) is essentially equivalent to the existence of an equivalent martingale measure. The problem of fair pricing of financial assets is then reduced to taking their expected value with respect to equivalent martingale measures.

We want in this paper to find the analog of the Fundamental Theorem of Asset Pricing for very general models of investment opportunities. Such opportunities

*CREST-ENSAE, 15, Bd Gabriel Péri, 92241 Malakoff Cedex, France and CERMSEM-Université de Paris I. Jouini is currently visiting the Stern School of Business at NYU, 44 W 4th St, NY,

†CREST, 15, Bd Gabriel Péri, 92241 Malakoff Cedex, France. 
are described in a deterministic setting by Cantor-Lippman (1983, 1995), DermodyRockafellar (1991, 1995) and Adler-Gale (1997). More precisely, we adopt a model where all investment opportunities are described by their cash flows; for instance, in such a model, the investment opportunity which consists in buying, in a perfect financial model, at date $s_{1}$ one unit of a risky asset, whose price process is given by $\left(S_{t}\right)_{t \geq 0}$ and selling at date $s_{2}$ with $s_{1} \leq s_{2}$ the unit bought, is described by a process $\left(\Phi_{t}\right)_{t \geq 0}$ which is null outside $\left\{s_{1}, s_{2}\right\}$ and which satisfies $\Phi_{s_{1}}=-S_{s_{1}}$ and $\Phi_{s_{2}}=S_{s_{2}}$. Our investment opportunities are assumed to be very general: they are not specifically related to a market model, like in the just mentioned example. The time horizon is not supposed to be finite. The framework is the one of continuous time. We don't assume that there exists a numéraire, enabling investors to transfer money from one date to another and even if such possibilities exist, we do not assume that the lending rate is equal to the borrowing rate or that we have possibilities to borrow.

We find in this very general model that the assumption of no-free lunch is essentially equivalent to the existence of a normalization process such that the "net present value" of any investment opportunity is nonpositive, i.e. there exists a process such that the "net present value" of the normalized cash flows is nonpositive. We emphasize that neither interest rate nor net present value are part of our model. As in Dermody-Rockafellar (1991, 1995) and Adler-Gale (1997), there is no externally given term structure, which would be needed if one were to apply the classical criterion of net present value; these notions arise however as a consequence of the assumption of no-arbitrage.

We then use this very general result for specific financial market models: perfect financial model, for which we prove the well-known characterization of the assumption of no-arbitrage given by the Fundamental Theorem of Asset Pricing in finite time, and a slightly different version in infinite time; but mainly financial models with imperfections like imperfections on the numéraire, proportional transaction costs, short sale constraints, convex cone constraints, etc., for which we generalize existing results.

Initial results on the Fundamental Theorem of Asset Pricing in the perfect case were achieved by Harrison-Kreps (1979), Harrison-Pliska (1979), Kreps (1981) and Duffie-Huang (1986). Various generalizations are now available in the literature: in Dalang, Morton and Willinger (1989), the problem was solved in the case of finite discrete time, by only using the assumption of no-arbitrage. For discrete infinite or continuous time, the notion of free lunch is needed; Schachermayer (1994) mainly deals with the case of discrete infinite time. Continuous time models are studied by (among others) Ansel-Stricker (1990), Delbaen (1992), Delbaen-Schachermayer (1994, 1998), Frittelli-Lakner (1994), Stricker (1990). In all these models, securities markets are assumed to be frictionless.

In the context of imperfect financial markets, Jouini-Kallal (1995a) characterize the assumption of no-free lunch in a model with proportional transaction costs and give fair pricing intervals for contingent claims in such a model. Cvitanic-Karatzas (1996) study the problem of hedging contingent claims, in continuous time, for a diffusion model (with one bond and one risky asset) with proportional transaction costs, and give a dual formula for the so-called super-replication price of a contingent claim (i.e. the minimum initial wealth needed to hedge the contingent claim or in other words, to obtain, through the investment opportunities available on the market, at least the contingent claim). Delbaen-Kabanov-Valkeila (1998) and Kabanov (1998) generalize this result to the multivariate case, in discrete as well as in continuous time, 
and with a semimartingale price process.

As for other imperfections, Jouini-Kallal (1995b) study the case of short sale constraints or shortselling costs with possibly different borrowing and lending rates. For convex constraints (and also with possible higher interest rates for borrowing), the dual formulation for the superreplication price is obtained in a diffusion framework in Cvitanic-Karatzas (1993). In a more general framework, the result is obtained in Föllmer-Kramkov (1997). Pham-Touzi (1996) consider the Fundamental Theorem of Asset Pricing with cone constraints, in a discrete and finite time setting and by only using the assumption of no-arbitrage. Brannath (1997) studies the same problem in the more general setting of convex constraints.

This paper generalizes Carassus-Jouini (1997), that considers discrete models, i.e. with finite time horizon, discrete time and finite number of states of the world at each time.

We generalize existing results in the following ways: first, we don't assume that there exists a numéraire available to investors and allowing them to transfer money from one date to another; this enables us to consider any type of friction on the numéraire like no borrowing, different borrowing and lending rates, bonds with default risk, etc., which have been barely studied, or simply to take into account the fact that all investors are not equal with regard to borrowing and lending, namely some investors may enjoy special borrowing facilities while others may not; second, we are lead to introduce a new notion of no-free lunch, which is similar to the "usual one" (with deterministic times) in finite time but does not exclude a free lunch at infinity and is therefore maybe more economically meaningful; last, we characterize the assumption of no-arbitrage (or more precisely of no-free lunch) for very general investments, which enables us to consider investment opportunities that are not necessarily related to a market model and, more interestingly, to generalize the results obtained for imperfect markets and to obtain them all in a unified way.

The paper is organized as follows: in section 2, we obtain, under a "reasonable" assumption, an analog of Yan (1980)'s result in more complex spaces than the ones usually considered. Indeed, since we are not allowed to transfer money from one date to another, we cannot consider net gains anymore; in all papers dealing with the Fundamental Theorem of Asset Pricing (with simple integrands), the assumption of no-arbitrage or no-free lunch essentially amounts to saying that the set $\operatorname{Lin}\left\{\theta_{s} \cdot\left(\bar{S}_{t}-\bar{S}_{s}\right)\right\}$, where $\theta$ describes all feasible strategies and $\bar{S}$ denotes the discounted underlying price process, contains no nonnegative nonnull random variable. Implicit in such an approach is the fact that there is an externally given term structure, enabling investors to borrow and lend money at the same rate. In our context with flows, we must use separation techniques in more complex spaces to obtain the Fundamental Theorem of Asset Pricing. We apply this characterization of the no-free lunch assumption to different market models with frictions in sections 3 and 4 .

All the proofs are in the Appendix.

\section{The Fundamental Theorem of Asset Pricing in a model with flows}

As we have sen in the Introduction, since we are not necessarily allowed to transfer wealth through time, we must consider more general spaces. We start by introducing these spaces. Then we describe our general model with flows. Finally, we obtain, 
through an analog of Yan's (1980) result, the characterization of the no free lunch assumption in such a model.

\subsection{The space $L_{P}^{1}\left(\Omega, \mathcal{M}_{b}\right)$}

For details about most notions introduced in this section, see Marle (1974) or DiestelUhl (1977).

We denote by $\mathcal{C}_{0}$ the set of continuous functions from $R$ to $R$ which converge to 0 at infinity; endowed with the uniform convergence topology, $\mathcal{C}_{0}$ is a Banach space. We denote by $\mathcal{M}_{b}$ the space of bounded Radon measures, i.e. the space of continuous linear functionals on $\mathcal{C}_{0}$; the space $\mathcal{M}_{b}$, endowed with the usual dual norm \|\|$_{\mathcal{M}_{b}}$ defined by $\|\mu\|_{\mathcal{M}_{b}} \equiv \sup \left\{|\mu(f)| ; f \in \mathcal{C}_{0} ;\|f\| \leq 1\right\}$ for all $\mu$ in $\mathcal{M}_{b}$, is a Banach space.

Fix a probability space $(\Omega, F, P)$. Let $\left(X,\|\|_{X}\right)$ be a Banach space. The set St $(\Omega, X)$ denotes the set of simple random variables, i.e., the set of random variables $f$ of the form $f=\sum_{i=1}^{m} a_{i} 1_{A_{i}}$ for some $m$ in $N^{*}, A_{i}$ in $F$ and $a_{i}$ in $X$. The set $M_{P}(\Omega, X)$ denotes the set of $P$-measurable random variables, i.e. the set of random variables $f$ such that there exists a sequence $\left(f_{n}\right)_{n \in N}$ in $S t(\Omega, X)$ for which $f=\lim _{n} f_{n}$ a.s. $P$. Then $L_{P}^{1}(\Omega, X)$ denotes the set of $P$-measurable random variables $f$ such that $\|f\|_{X}$ belongs to $L^{1}(\Omega, R)$, i.e. such that $\|f\|_{L_{P}^{1}(\Omega, X)} \equiv E^{P}\left[\|f\|_{X}\right]<\infty$.

Denote by $\left(X^{\prime},\|\|_{X^{\prime}}\right)$ the dual space of $X$, endowed with the dual norm. We know that $\left(X^{\prime},\|\|_{X^{\prime}}\right)$ is itself a Banach space. We define the space $\mathcal{L}_{*}^{\infty}\left(\Omega, P, X^{\prime}\right)$ to be the set of random variables $g: \Omega \rightarrow X^{\prime}$, for which

$$
\begin{gathered}
\langle g, e\rangle: \omega \rightarrow\langle g(\omega), e\rangle \text { is measurable for all } e \text { in } X \text { and } \\
\|g\|_{\mathcal{L}_{*}^{\infty}\left(\Omega, P, X^{\prime}\right)} \equiv \inf \left\{M \geq 0,\|g\|_{X^{\prime}} \leq M \quad P \text { a.s. }\right\}<\infty .
\end{gathered}
$$

Then the set $L_{*}^{\infty}\left(\Omega, P, X^{\prime}\right)$ is defined to be the set of equivalence classes $g^{\bullet}$ of elements of $\mathcal{L}_{*}^{\infty}\left(\Omega, P, X^{\prime}\right)$, where $f \sim g \Leftrightarrow\langle f, e\rangle=\langle g, e\rangle P$ a.s. for all $e$ in $X$. We consider the norm \|\|$_{L_{*}^{\infty}\left(\Omega, P, X^{\prime}\right)}$ given by $\|g\|_{L_{*}^{\infty}\left(\Omega, P, X^{\prime}\right)}=\inf _{g \in g^{\bullet}}\|g\|_{\mathcal{L}_{*}^{\infty}\left(\Omega, P, X^{\prime}\right)}$ for all $g \in L_{*}^{\infty}\left(\Omega, P, X^{\prime}\right)$.

It is shown in Schwartz (1974) that the dual space of $L_{P}^{1}(\Omega, X)$ is the space $L_{*}^{\infty}\left(\Omega, P, X^{\prime}\right)$. More precisely, there is an isomorphism between $L_{*}^{\infty}\left(\Omega, P, X^{\prime}\right)$ and $\left[L_{P}^{1}(\Omega, X)\right]^{\prime}$ that associates with any random variable $g$ in $L_{*}^{\infty}\left(\Omega, P, X^{\prime}\right)$ the continuous linear functional $\Psi$ on $L_{P}^{1}(\Omega, X)$ given by

$$
\Psi: f \rightarrow \Psi(f)=E\left[\langle f, g\rangle_{X, X^{\prime}}\right] \quad \text { for all } f \text { in } L_{P}^{1}(\Omega, X)
$$

where $\langle f, g\rangle_{X, X^{\prime}}: \omega \mapsto\langle f(\omega), g(\omega)\rangle_{X, X^{\prime}}$. Notice then that $L_{*}^{\infty}\left(\Omega, P, X^{\prime}\right)$ is a Banach space.

Let $\Gamma$ denote the set of random variables $\gamma$ from $\Omega$ to $\mathcal{M}_{b}$ of the form $\gamma=$ $\sum_{i=1}^{m} \gamma_{t_{i}} \delta_{t_{i}}$ i.e. $\gamma: \omega \mapsto \gamma_{t_{1}}(\omega) \delta_{t_{1}}+\ldots+\gamma_{t_{m}}(\omega) \delta_{t_{m}}$ with $\gamma_{t_{i}}$ in $L^{1}(\Omega, R)$ for all $t_{i} \geq 0$. We should write $\gamma=\sum_{i=1}^{m_{\gamma}} \gamma_{t_{i}^{\gamma}} \delta_{t_{i}^{\gamma}}$ but we will omit the index $\gamma$ for the simplicity of the notations.

We have the following

Lemma 2.1. The set $\Gamma$ is included in $L_{P}^{1}\left(\Omega, \mathcal{M}_{b}\right)$ and for all $\gamma$ in $\Gamma, \gamma=\sum_{i=1}^{m} \gamma_{t_{i}} \delta_{t_{i}}$, $\|\gamma\|_{L_{P}^{1}\left(\Omega, \mathcal{M}_{b}\right)}=\sum_{i=1}^{m}\left\|\gamma_{t_{i}}\right\|_{L^{1}(\Omega, R)}$.

Let $\Gamma_{+}\left(\right.$resp. $\left.\Gamma_{-}\right) \equiv\left\{\gamma \in \Gamma ; \gamma=\sum_{i=1}^{m} \gamma_{t_{i}} \delta_{t_{i}} ; \gamma_{t_{i}} \geq 0\right.$ (resp. $\left.\leq 0\right) P$ a.s. $\}$. 


\subsection{The model}

We consider a model in which agents face investment opportunities described by their cash flows. A probability space $(\Omega, F, P)$ is specified and fixed. The set $\Omega$ represents all possible states of the world. An information structure, which describes how information is revealed to investors, is given by a filtration $\left(F_{t}\right)_{t \in \mathbf{T}}$ satisfying the "usual conditions" and such that $F_{0}=\{\emptyset, \Omega\}$. In finite discrete time, we take $\mathbf{T}=\{1, \ldots, d\}$, in infinite discrete time $\mathbf{T}=N$ and in infinite continuous time $\mathbf{T}=R_{+}$. We model investment opportunities which are available to investors in the following way.

Definition 2.2. An investment is an $\left(F_{t}\right)_{t \in \mathbf{T}^{-} \text {-adapted process }} \Phi=\left(\Phi_{t}\right)_{t \in \mathbf{T}}$, null outside a finite number of dates, i.e. there exists $\left(t_{1}^{\Phi}, \ldots, t_{N}^{\Phi}\right)$ such that $\Phi_{t}=0$ for all $t \notin\left(t_{i}^{\Phi}\right)_{i=1}^{N}$, and such that $\Phi_{t}$ is in $L^{1}\left(\Omega, F_{t}, P\right)$ for all $t$ in $\mathbf{T}$.

We consider a convex cone $J$ of investment opportunities: this amounts to saying that an investor has a right to subscribe to (a finite number of) different investment plans and that he can decide at the starting date of any investment opportunity which amount of this particular investment he wants to buy. We shall see in sections 3 and 4 , with specific examples, that we are lead to consider convex cones in order to take into account the fact that investors are not necessarily able to sell an investment plan (see for instance the case of short sale constraints or transaction costs).

We don't specify the elements of $J$ so far. We consider any investment $\Phi=\left(\Phi_{t}\right)_{t \in \mathbf{T}}$ as a random variable from $\Omega$ to the set of discrete bounded Radon measures of the form

$$
\Phi=\sum_{t \geq 0} \Phi_{t} \delta_{t}
$$

where $\Phi_{t}=0$ for $t \notin\left(t_{i}^{\Phi}\right)_{i=1}^{N}$, i.e. as an element of $\Gamma$.

We introduce the following assumption.

Assumption $A$ : there exists a sequence $d=\left(d_{n}\right)_{n \geq 0}$ such that for all $t^{*} \geq 0$, for all $B_{t^{*}}$ in $F_{t^{*}}$ of positive probability, there exists $\Phi$ in $J$ of the form $\Phi_{t^{*}}=0$ outside $B_{t^{*}}$, $\Phi_{t}=0$ for all $t<t^{*}, \Phi_{t} \geq 0$ for all $t>t^{*}$, and there exists $d_{n} \in d, P\left[\Phi_{d_{n}}>0\right]>0$.

In words, if a convex cone $J$ of available investments satisfies Assumption A, this means that there exists a sequence of trading dates such that, for all date and for all event at that date, there exists an investment plan in our admissible set of investment opportunities that starts at that date and in that event, that can take any value at that date and in that event but that is nonnegative after that date and nonnull at one date belonging to the above mentioned sequence of dates. Roughly, Assumption A corresponds to the possibility of transferring "some money" from any date and event to some particular date. This assumption is not too restrictive: it is satisfied if we can buy at every date and event a bond with a given maturity even if this bond is defaultable and even if there is no secondary market for that bond (i.e. we have to wait until the maturity in order to recover any money with a positive probability, which may be different from 1); this includes market models with frictions on the numéraire like no borrowing, different borrowing and lending rates, bonds with default risk, different borrowing facilities among investors. More generally, it is satified if there is at least one asset whose price cannot be negative (which is usually the case for stocks or for options, defaultable bonds, etc.). Notice that it is satisfied in all cases of market 
models with frictions that we have mentioned in the introduction and that we shall consider more precisely in sections 3 and 4 .

We now come to the notion of no-arbitrage.

Definition 2.3. There is no arbitrage opportunity for $J$ if and only if $J \cap \Gamma_{+}=\{0\}$.

Let us check that this definition corresponds to the usual notion of no-arbitrage, i.e. an impossibility to have access to an investment that yields a positive gain in some circumstances without a countervailing threat of loss in other circumstances. In our framework, an arbitrage opportunity would consist in a nonnegative nonnull investment. And $\Phi=\left(\Phi_{t}\right)_{t \in \mathbf{T}}$ is a nonnegative nonnull investment process if and only if $\Phi: \omega \mapsto \sum_{i=1}^{N} \Phi_{t_{i}^{\Phi}}(\omega) \delta_{t_{i}^{\Phi}}$, which is in $L_{P}^{1}\left(\Omega, \mathcal{M}_{b}\right)$, belongs to $\Gamma_{+}$and is not null.

It is easy to see that the notion of no-arbitrage introduced in Definition 2.3 can be written in the form $\left(J-\Gamma_{+}\right) \cap \Gamma_{+}=\{0\}$. A free lunch denoting the possibility of getting arbitrarily close to an arbitrage opportunity, we introduce the following

Definition 2.4. There is no free lunch for $J$ if and only if $\overline{\left(J-\Gamma_{+}\right)} \cap \Gamma_{+}=\{0\}$, where the bar denotes the closure in $L_{P}^{1}\left(\Omega, \mathcal{M}_{b}\right)$.

Notice that if there is a numéraire, the assumption $\overline{\left(J-\Gamma_{+}\right)} \cap \Gamma_{+}=\{0\}$, where the bar denotes the closure in $L_{P}^{1}\left(\Omega, \mathcal{M}_{b}\right)$, is less restrictive than the "usual" assumption of no-free lunch (with deterministic times). The difference between the two notions lies in the fact that the nonnegative nonnull random variable that we can "almost reach" with admissible investments "is" at a finite date for the new notion whereas it can be "at infinity" for the usual notion. The notion of absence of free lunch adopted in this paper does not exclude free lunches "at infinity" and can therefore be considered as more economically meaningful.

\subsection{Characterization of the no-arbitrage assumption in a model with flows}

By adapting the proof of Yan (1980), we obtain, under Assumption A, the following Fundamental Theorem of Asset Pricing in our general framework with flows.

Theorem 2.5. Let $J$ denote a convex cone of investments satisfying Assumption A. There is no free lunch for $J$ if and only if there exists a process $g=\left(g_{t}\right)_{t \in \mathbf{T}}$ satisfying for all $t$ in $\mathbf{T}, P\left(0<g_{t}<M\right)=1$ for some $M$ in $R_{+}^{*}$, and such that

$$
E\left[\sum_{t \in \mathbf{T}} g_{t} \Phi_{t}\right] \leq 0 \quad \text { for all } \Phi=\left(\Phi_{t}\right)_{t \in \mathbf{T}} \in J
$$

Moreover, the process $g$ can be taken $\left(F_{t}\right)_{t \in \mathbf{T}^{-}}$adapted.

In the case where the set of investment opportunities is related to a countable set of dates, which is the case in finite (resp. infinite) discrete time where $\mathbf{T}=\{1, \ldots, d\}$ (resp. $\mathbf{T}=N$ ), then Assumption $A$ is not needed to obtain Theorem 2.5.

Corollary 2.6. Let $J$ denote a convex cone of investments. In finite or infinite discrete time, there is no free lunch for $J$ if and only if there exists a P-essentially bounded process $g=\left(g_{t}\right)_{t \in \mathbf{T}}$ such that inequation 2.1 holds. 
So, in continuous time, starting from the assumption of no free-lunch in a very general model with flows, without any assumption on the existence of a numéraire, but under Assumption $A$ (for which we have given an interpretation and that will reveal to be suited to our market frictions), we have proved the existence of a "discount process" such that, using this process as a deflator, all available investments have non-positive present value; this means that there exists a term structure such that the market consisting of the primitive investment opportunities and of the additional borrowing and lending facilities is still "arbitrage-free". Besides, the existence of such a discount process prevents from any arbitrage opportunity. In other words, there is no free lunch for a convex cone of available investments satisfying Assumption A if and only if a given convex set of "admissible" discount processes is non-void. We recall that Assumption $A$ is not needed if we deal with discrete, possibly infinite, time.

We have seen that the "discount process" can be taken adapted to our filtration. We have obtained so far no other regularity conditions (such as right-continuity); we shall see in the following section that under some regularity conditions on the investments, we obtain regularity properties on the "discount process" itself. Besides, the taking into account of frictions on the numéraire will enable us to better understand the nature of these admissible discount processes.

Since most market models with frictions can fit in the model with flows for a specific convex cone of available investments, our model provides a unified framework for the study of the characterization of the absence of free lunch in such imperfect market models. Notice however that economies with fixed transactions costs do not fall in our model with flows, since the set of all available investments is not a cone.

\section{Application to market models with frictions on the numéraire}

In this section, we still consider a general framework with flows, but we introduce a numéraire, possibly submitted to constraints. This enables us to give a better interpretation of the "admissible" discount processes found in the previous section. Moreover, we obtain a characterization of the no-free lunch assumption in general models with flows with possible frictions on the numéraire. Since market imperfections like convex cone constraints, proportional transaction costs, etc. can fit in our model with flows, our approach will enable us in the next section to characterize the absence of free lunch in these imperfect models, when there are, in addition, imperfections on the numéraire.

We introduce a few notations. Let $\mathcal{G}$ denote the set of all (equivalence classes) of processes $g=\left(g_{t}\right)_{t>0}$, such that for all $t \in R_{+}, 0<g_{t} \leq M^{g}$ for some scalar $M^{g}$. For a convex cone $\bar{K}$ in $\Gamma$, let $\mathcal{G}_{K}$ denote the convex set of processes $g \in \mathcal{G}$ such that $E\left[\sum_{t \geq 0} g_{t} \Phi_{t}\right] \leq 0$ for all $\Phi \in K$.

\subsection{With a "perfect" numéraire}

We assume that there is a perfect numéraire, i.e. that there are possibilities to transfer money, without friction, from one date to another. Let $S^{0}=\left(S_{t}^{0}\right)_{t \geq 0}$ denote a positive, adapted (numéraire) process such that for all $\left(t_{1}, t_{2}\right) \in R_{+}^{2}$, for all $\theta \in$ $L^{\infty}\left(\Omega, F_{t_{1} \wedge t_{2}}, P\right)$, the process denoted by $\Phi^{\left(0 ; \theta, t_{1}, t_{2}\right)}$ and given by

$$
\Phi_{t}^{\left(0 ; \theta, t_{1}, t_{2}\right)}=\theta\left[-S_{t_{1}}^{0} 1_{t=t_{1}}+S_{t_{2}}^{0} 1_{t=t_{2}}\right] \quad \text { for all } t \geq 0
$$


belongs to $\Gamma$. Notice that this is equivalent to $S_{t}^{0} \in L^{1}\left(\Omega, F_{t}, P\right)$ for all $t \geq 0$. Let $J^{\text {num }}$ denote the convex cone generated by a given convex cone $J$ in $\Gamma$ and all the investments $\Phi^{\left(0 ; \theta, t_{1}, t_{2}\right)}$. The set $J^{\text {num }}$ corresponds to all available investments in a model with flows, where agents can invest in a perfect numéraire. Notice that $J^{\text {num }}$ satisfies Assumption A so that, according to Theorem 2.5, there is no free lunch in

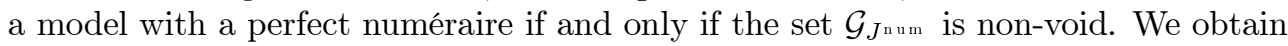
the following characterization of the set of admissible discount processes.

Lemma 3.1. The set $\mathcal{G}_{J^{\text {num }}}$ consists of all processes $g \in \mathcal{G}_{J}$ such that $g S^{0}$ is a $P$ martingale for $\left(F_{t}\right)_{t \geq 0}$.

This means that if we introduce a term structure given by a numéraire $S^{0}$, then in the absence of free lunch, the process $g$ must be equal to $\frac{1}{S 0}$ multiplied by a positive martingale (which is the stochastic analog of a (positive) constant function). Up to a martingale, the process $g$ gives us the possible term structures, i.e. which would be compatible with the assumption of no-free lunch. Note that the process $g S^{0}$ admits a right-continuous left-limited (RCLL) modification, so that if $1 / S^{0}$ is RCLL, then $g$ itself admits a RCLL modification.

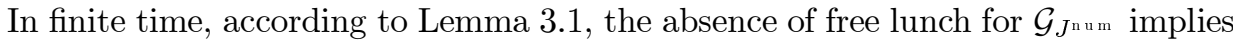
that there exists an equivalent probability measure under which the net present value (using $S^{0}$ as a deflator) of any available investment is nonpositive, i.e. there exists a probability measure $Q \sim P$, such that $E^{Q}\left[\sum_{t \geq 0} \frac{\Phi_{t}}{S_{t}^{0}}\right] \leq 0$ for all $\Phi \in J$. In infinite time, the situation is somewhat different.

Corollary 3.2. $\quad$ 1. If $S^{0}$ is uniformly integrable, then there is no free lunch for $J^{\text {num }}$ if and only if there exists an absolutely continuous probability measure $\bar{P}$ on $(\Omega, F, P)$, such that $\left(\frac{1}{S_{t}^{0}} E\left[\frac{d \bar{P}}{d P} \mid F_{t}\right]\right) \in \mathcal{G}$ and $E^{\bar{P}}\left[\sum_{t \geq 0} \frac{\Phi_{t}}{S_{t}^{0}}\right] \leq 0$ for all investment $\Phi$ in $J$.

2. If..., there is no free lunch for $J^{\text {num }}$ if and only if there exists a probability $\bar{P}$ on $(\Omega, F)$, such that $\left(\frac{1}{S_{t}^{0}} E\left[\frac{d \bar{P}}{d P} \mid F_{t}\right]\right) \in \mathcal{G}$ and $E^{\bar{P}}\left[\sum_{t \geq 0} \frac{\Phi_{t}}{S_{t}^{0}}\right] \leq 0$ for all investment $\Phi$ in $J$.

Compared with the "classical case" with a perfect numéraire constantly equal to 1 (see e.g. Stricker (1990)), in the infinite time framework, we only find an absolutely continuous probability measure, whose restricted density to all $F_{t}$ is positive, instead of an equivalent probability measure and this is due to the fact that, as mentioned in section 2, our assumption of no-free lunch is less restrictive than the "usual" one, since it does not exclude free lunches at infinity.

We now turn to cases where the numéraire is subject to some constraints.

\subsection{With lending and borrowing opportunities}

Let $S^{0}=\left(S_{t}^{0}\right)_{t>0}$ and $S^{1}=\left(S_{t}^{1}\right)_{t>0}$ denote two positive, right-continuous and adapted processes with $S_{0}^{0}=S_{0}^{\prime 0}=1$, such that for all $\left(t_{1}, t_{2}\right) \in R_{+}^{2}$ with $t_{1} \leq t_{2}$, for all $\theta \in L_{+}^{\infty}\left(\Omega, F_{t_{1}}, P\right)$, the processes denoted by $\Phi^{\left(0 ; \theta, t_{1}, t_{2}\right)}$ and $\Phi^{\left(1 ; \theta, t_{1}, t_{2}\right)}$ and given by

$$
\begin{aligned}
& \Phi_{t}^{\left(0 ; \theta, t_{1}, t_{2}\right)}=\theta\left[-S_{t_{1}}^{0} 1_{t=t_{1}}+S_{t_{2}}^{0} 1_{t=t_{2}}\right] \quad \text { for all } t \geq 0 \\
& \Phi_{t}^{\left(1 ; \theta, t_{1}, t_{2}\right)}=\theta\left[S_{t_{1}}^{1} 1_{t=t_{1}}-S_{t_{2}}^{1} 1_{t=t_{2}}\right] \quad \text { for all } t \geq 0
\end{aligned}
$$


belong to $\Gamma$. Let $J^{\mathrm{lb}}$ denote the convex cone generated by a given convex cone $J$ in $\Gamma$ and all the investments $\Phi^{\left(0 ; \theta, t_{1}, t_{2}\right)}$ and $\Phi^{\left(1 ; \theta, t_{1}, t_{2}\right)}$. The set $J^{\mathrm{lb}}$ corresponds to all available investments in a market model where agents have lending and borrowing opportunities, but not in the same conditions. Notice that $J^{\mathrm{lb}}$ satisfies Assumption $A$ so that, according to Theorem 2.5, there is no free lunch in a model with such constraints on the lending and borrowing opportunities if and only if the set $\mathcal{G}_{J^{1 \mathrm{~b}}}$ is non-void. We obtain the following characterization of the set $\mathcal{G}_{J^{\mathrm{b}}}$.

Lemma 3.3. The set $\mathcal{G}_{J^{l b}}$ consists of all processes $g \in \mathcal{G}_{J}$ such that $g S^{0}$ is a $P$ supermartingale and $g S^{1}$ is a $P$-submartingale for $\left(F_{t}\right)_{t \geq 0}$.

We now introduce additional conditions on the processes $S^{0}$ and $S^{1}$.

(C1) For all $\left(t, t_{1}, t_{2}\right)$ with $t \leq t_{1} \leq t_{2}$, for all $A \in F_{t_{1}}$, the processes $\Phi\left(k ; \frac{1 A}{S_{t}^{k}, t_{1}, t_{2}}\right) \in$ $J^{\mathrm{lb}}$ for $k=0,1$.

(C2) For all $s \leq t, S_{t}^{0} / S_{s}^{0} \leq S_{t}^{1} / S_{s}^{1}$.

(C3) The processes $S^{0}$ and $S^{1}$ can be written in the form $S^{0}=\exp \int_{0}^{*} r_{s}^{0} d s$ and $S^{1}=\exp \int_{0}^{\cdot} r_{s}^{1} d s$ for some bounded and progressively measurable processes $r^{0}$ and $r^{1}$.

Condition (C1) is an integrability condition; it is satisfied if for all $t \geq 0,1 / S_{t}^{0}$ as well as $1 / S_{t}^{1}$ are bounded. Condition (C2) essentially means that the "borrowing rate" is greater than or equal to the "lending rate". Condition (C3) means that the processes $S^{0}$ and $S^{1}$ are associated to interest rates, with a possible spread. Notice that contrarily to Jouini-Kallal (1995 b), we do not suppose $r^{0} \leq r^{1}$, since we get it as a consequence of the absence of free lunch.

Lemma 3.4. If there is no free lunch for $J^{l b}$ and if Condition (C3) is satisfied, then for all $s \geq 0, r_{s}^{0} \leq r_{s}^{1}$ and Conditions $(C 1)$ and $(C 2)$ are satisfied .

We now obtain more specific characterizations of the set $\mathcal{G}_{J^{\mathrm{lb}}}$ under the different conditions.

Lemma 3.5. 1. Under Condition (C1), any $g \in \mathcal{G}_{J^{1 b}}$ admits a right-continuous modification.

2. Under Conditions $(C 1)$ and $(C 2)$, the set $\mathcal{G}_{J^{l b}}$ consists of all processes $g \in \mathcal{G}_{J}$ for which for all $s \geq 0$, there exists a process $\left(Z_{t}^{s}\right)_{t \geq s}$ satisfying $S_{t}^{0} / S_{s}^{0} \leq Z_{t}^{s} \leq$ $S_{t}^{1} / S_{s}^{1}$ for all $t \geq s$ such that $g Z^{s}$ is a (right-continuous) martingale for $\left(F_{t}\right)_{t>s}$.

3. Under Condition (C3), the set $\mathcal{G}_{J^{l b}}$ consists of all processes $g \in \mathcal{G}_{J}$ for which there exists a process $Z \equiv \exp \int_{0}^{\cdot} r_{s} d s$ for some bounded and progressively measurable processes $r$ satisfying $r_{s}^{0} \leq r_{s} \leq r_{s}^{1}$ for all $s \geq 0$ such that $g Z$ is a (right-continuous) martingale.

Part 1 says that the right continuity of the borrowing and lending processes induce the right-continuity of the admissible discount processes. Part 2 says up to a martingale, the returns of the admissible discount processes lie between the returns of the lending and borrowing processes; notice that if $1 / S^{0}$ is LL, than any admissible discount process admits a LL modification. Part 3 says that if the lending and borrowing processes are associated to interest rates, then so are the admissible discount processes. 


\subsection{With lending opportunities only}

With the notations adopted in the previous subsection, let $J^{\mathrm{b}}$ denote the convex cone generated by a given convex cone $J$ in $\Gamma$ and all the investments $\Phi^{\left(0 ; \theta, t_{1}, t_{2}\right)}$. The set $J^{\mathrm{b}}$ corresponds to all available investments in a market model where investors have lending opportunities, but not necessarily borrowing opportunities. Notice that $J^{\mathrm{b}}$ satisfies Assumption $A$ so that there is no free lunch in a model with no borrowing if and only if the set $\mathcal{G}_{J^{\mathrm{b}}}$ is non-void.

Lemma 3.6. The set $\mathcal{G}_{J^{b}}$ consists of all processes $g \in \mathcal{G}_{J}$ such that $g S^{0}$ is a supermartingale.

We can now turn to imperfections not only involving the numéraire.

\section{Application to other market models with frictions}

\subsection{Models with dividends}

We consider a model of financial market consisting of $N$ financial assets, possibly paying dividends to their holders. We denote by $\left(S^{k}\right)_{1<k<N}$ the adapted price processes of the securities and by $\left(D^{k}\right)_{1 \leq k<N}$ the associated (possibly null) adapted dividends processes. We assume that the dividends are discrete, i.e. that there is no dividend paid outside a countable set of dates $\left(u_{n}^{k}\right)_{n>0}$ : the random variable $D_{t}^{k}$ corresponds to the dividends paid by the security $k$ at time $t$ and we have $D_{t}^{k}=0$ for $t \notin\left(u_{n}^{k}\right)_{n \geq 0}$. We treat $S_{t}^{k}$ by convention as the post-dividends market value of the security $k$ at time $t$. In other words, if the dividend process jumps at time $t$, the market value $S_{t}^{k}$ reflects the jump as already having been paid out, or $S_{t}^{k}$ is ex-dividend.

For $1 \leq k \leq N$, for all $\left(t_{1}, t_{2}\right) \in R_{+}^{2}$ with $t_{1} \leq t_{2}$, and for all $A \in F_{t_{1}}$, we assume that the process $\Phi^{\left(k ; A, t_{1}, t_{2}\right)}$ given by

$$
\Phi_{t}^{\left(k ; A, t_{1}, t_{2}\right)}=1_{A}\left[-S_{t_{1}}^{k} 1_{t=t_{1}}+D_{t}^{k} 1_{t_{1}<t \leq t_{2}}+S_{t_{2}}^{k} 1_{t=t_{2}}\right] \quad \text { for all } t \geq 0
$$

belongs to $\Gamma$ and we let $J_{\text {Div }}$ denote the linear space generated by all these investments. We suppose that Assumption $A$ is satisfied. Using Theorem 2.5, we get that

Theorem 4.1. There is no free lunch in the model with dividends (or equivalently for $\left.J_{D i v}\right)$ if and only if there exists a process $g=\left(g_{t}\right)_{t \geq 0} \in \mathcal{G}$ such that for all $t_{1} \leq t_{2}$

$$
E\left[g_{t_{2}}\left(S_{t_{2}}+\sum_{t_{1}<t \leq t_{2}} g_{t} D_{t}\right) \mid F_{t_{1}}\right]=g_{t_{1}} S_{t_{1}} .
$$

Note that in the perfect case with no dividend, a finite time horizon, and a perfect numéraire (constantly equal to one), we obtain the "classical" Fundamental Theorem of Asset Pricing, which asserts that there is no free lunch if and only if there exists an equivalent probability measure with bounded density, which makes the (discounted) price process of the traded securities a martingale. 


\subsection{Convex cone constraints and applications to short sale constraints}

We consider now a model of financial market where the quantities of the $N$ different risky assets held by investors are constrained to lie in a convex cone $\mathcal{C}$ (in $R^{N}$ ). Notice that this situation includes the one with no constraint $\left(\mathcal{C}=R^{N}\right)$.

We denote by $\left(S^{k}\right)_{1 \leq k \leq N}$ the adapted price process of the risky assets. For $1 \leq k \leq N$, for all $\left(t_{1}, t_{2}\right) \in R_{+}^{2}$, and for all $A \in F_{t_{1} \wedge t_{2}}$, we assume that the process $\Phi^{\left(k ; A, t_{1}, t_{2}\right)}$ given by

$$
\Phi_{t}^{\left(k ; A, t_{1}, t_{2}\right)}=1_{A}\left[-S_{t_{1}}^{k} 1_{t=t_{1}}+S_{t_{2}}^{k} 1_{t=t_{2}}\right] \quad \text { for all } t \geq 0
$$

belongs to $\Gamma$. Let $\Phi^{\left(A, t_{1}, t_{2}\right)}=\left(\Phi^{\left(k ; A, t_{1}, t_{2}\right)}\right)_{1 \leq k \leq N}$. Let $J_{\text {Vex }}$ denote the following set

$$
\left\{x \cdot \Phi^{\left(A, t_{1}, t_{2}\right)} ; x \in \mathcal{C},\left(t_{1}, t_{2}\right) \in R_{+}^{2}, A \in F_{t_{1} \wedge t_{2}}\right\}
$$

The set $J_{\text {Vex }}$ corresponds to all available investments in a model where portfolios are constrained to lie in a convex cone. Let $\mathcal{C}^{0}=\left\{y \in R^{N} ; y \cdot x \leq 0\right.$, for all $\left.x \in \mathcal{C}\right\}$. In the unconstrained case, we have $\mathcal{C}^{0}=\{0\}$. We get the following characterization of the set $\mathcal{G}_{J_{\mathrm{Vex}}}$.

Theorem 4.2. The set $\mathcal{G}_{J_{V e x}}$ consists of all processes $g \in \mathcal{G}$ such that for all $t_{1} \leq t_{2}$, $E\left[g_{t_{2}} S_{t_{2}}-g_{t_{1}} S_{t_{1}} \mid F_{t_{1}}\right] \in \mathcal{C}^{0}$.

We can apply this result to market models with short sale constraints. We consider a model of financial markets where two sorts of securities can be traded. Short selling the first type of securities is not allowed, i.e. they can only be held in nonnegative amounts, whereas the second type of securities can only be held in nonpositive amounts. The model includes situations where holding negative amounts of a security is possible but costly as well as situations where some (or all) securities are not subject to any constraints, since we may include a security twice in the model, in the first and in the second set of securities. This model has been studied in Jouini-Kallal (1995b), in finite time, for price processes which are in $L^{2}(\Omega, F, P)$ and right-continuous.

This situation falls in our framework with convex cone constraints for

$$
\mathcal{C}_{s}=\left\{x=\left(x_{1}, \ldots, x_{N}\right) \in R^{N} ; x^{k} \geq 0 \text { for } k \leq j ; x^{k} \leq 0 \text { for } k>j\right\}
$$

in the case where the first $j$ assets cannot be sold short. Then

$$
\mathcal{C}_{s}^{0}=\left\{y=\left(y_{1}, \ldots, y_{N}\right) \in R^{N} ; y^{k} \leq 0 \text { for } k \leq j \text { and } y^{k} \geq 0 \text { for } k \geq j\right\} .
$$

We then easily obtain the following characterization of the set $\mathcal{G}_{J_{\mathrm{S}}}$, where $J_{\mathrm{S}}$ is given by $J_{\text {Vex }}$ for $\mathcal{C}=\mathcal{C}_{s}$.

Corollary 4.3. The set $\mathcal{G}_{J_{S}}$ of admissible discount processes in the case with short sale constraints consists of all processes $g \in \mathcal{G}$, such that for all security $k \leq j$ that cannot be sold short, $g S^{k}$ is a supermartingale, and for all security $k>j$ that can only be sold short, $g S^{k}$ is a submartingale.

\subsection{Proportional transaction costs}

We consider a model of financial market where the securities are subject to bid-ask spreads: at each date, there is not a unique price for a security but an ask price, 
at which investors can buy the security and a bid price, at which they can sell the security. Notice that this model includes situations where there is a unique price process $S$ and where the transaction cost remains constant overtime, i.e. situations where at each time $t \geq 0$, investors must pay $S_{t}(1+c)$ for some positive constant $c$ to buy the security and receive $S_{t}(1-c)$ when selling it.

Such a model with transaction costs has been studied in Jouini-Kallal (1995a) using simple trading strategies like in Harrison-Kreps (1979), assuming that the time horizon is finite (equal to $T$ ), that there exists a numéraire process (identically equal to one) and that for all $t \geq 0$, the bid and ask prices at date $t$ are in $L^{2}\left(\Omega, F_{t}, P\right)$. We intend here to obtain the result of Jouini-Kallal (1995a) by using our approach with general flows, and to extend it mainly to the case with imperfections on the numéraire and to infinite time.

More precisely, we consider $(N-1)$ securities and for $2 \leq k \leq N$, we let $\left(S_{t}^{k}\right)_{t \geq 0}$ and $\left(S_{t}^{\prime k}\right)_{t \geq 0}$ denote respectively the adapted ask and bid price process. We assume that, for each $k$, the processes $S^{k}$ and $S^{\prime k}$ are right-continuous and of class $\mathcal{D}_{f}$, i.e. that the families $\left\{S_{\tau}\right\}_{\tau \in \mathcal{S}_{f}}$ and $\left\{S_{\tau}^{\prime}\right\}_{\tau \in \mathcal{S}_{f}}$ are uniformly integrable, where $\mathcal{S}_{f}$ denotes the collection of stopping times of $\left(F_{t}\right)_{t \geq 0}$, taking only a finite number of values in $[0,+\infty]$. Let $S_{t, \infty}^{f}$ denote the collection of stopping times $\tau$ in $\mathcal{S}_{f}$ such that $\tau \geq t$ a.s. $P$.

For each $k$, for any stopping times $\tau_{1}$ and $\tau_{2}$ in $\mathcal{S}_{f}$, we consider the process $\Phi^{\left(k ; \tau_{1}, \tau_{2}\right)}$ given by

$$
\Phi_{t}^{\left(k ; \tau_{1}, \tau_{2}\right)}=-S_{\tau_{1}}^{k} 1_{t=\tau_{1}}+S_{\tau_{2}}^{\prime k} 1_{t=\tau_{2}} \quad \text { for all } t \geq 0 .
$$

It is easy to see that for each $k$, for any stopping times $\tau_{1}$ and $\tau_{2}$ in $S^{f}$, the process $\Phi^{\left(k ; \tau_{1}, \tau_{2}\right)}$ is an investment as defined in Definition 2.2 and we define the set $J_{\mathrm{Costs}}$ as the convex cone generated by all these investments.

With the notations adopted in the subsection 3.2, we make the additional assumption that there exist two processes $S^{0}$ and $S^{1}$ satisfying Condition (C1) and we denote by $J_{\mathrm{C}}^{\mathrm{lb}}$ the convex cone generated by $J_{\mathrm{Costs}}$ and all the investments $\Phi^{\left(0 ; \theta, t_{1}, t_{2}\right)}$ and $\Phi^{\left(1 ; \theta, t_{1}, t_{2}\right)}$. We denote by $\mathcal{G}_{\mathrm{lb}}$ the set of processes $g \in \mathcal{G}$ such that $g S^{0}$ is a $P$-supermartingale and $g S^{1}$ is a $P$-submartingale for $\left(F_{t}\right)_{t>0}$, according to Lemma $3.3, \mathcal{G}_{\mathrm{lb}}$ is the set of discout processes compatible with the absence of free lunch for the convex cone generated by all invesments related to the borrowing and lending processes.

Notice that the no-arbitrage assumption implies that the bid price process lies below the ask price process. We then have the following

Theorem 4.4. There is no free lunch for $J_{\text {Costs }}^{l b}$ if and only if there exist an adapted process $g=\left(g_{t}\right)_{t>0} \in \mathcal{G}_{1 b}$ and some adapted price process $\tilde{S}$ lying between the bid and ask price processes such that $g \tilde{S}$ is a (right-continuous) $P$-martingale for $\left(F_{t}\right)_{t \geq 0}$.

If we now suppose that there is a perfect numéraire $S^{0} \equiv 1$, we get the result obtained by Jouini-Kallal (1995a) in a finite time setting, i.e. there is no free lunch with proportional transaction costs if and only if there exists an equivalent probability measure, with bounded density (in $L^{2}\left(\Omega, F_{T}, P\right)$ in the setting of Jouini-Kallal (1995a)) that transforms some process between the bid and the ask price processes into a martingale. Theorem 4.4 enables to consider market models with proportional transaction costs and imperfections on the numéraire, in an infinite time setting. 


\section{Conclusion}

We have characterized the assumption of no-arbitrage in financial markets, where any investment opportunity is described by the cash flows that it generates; the absence of free lunch is equivalent to the existence of a normalization process such that the "net present value" of any investment is nonpositive, i.e. there exists a process such that the "net present value" of the normalized cash flows is nonpositive. We have then applied this very general result to specific financial market models and mainly financial models with imperfections like imperfections on the numéraire, which had been barely studied, but also proportional transaction costs, short sale constraints, convex cone constraints, for which we have generalized, in a unified way, existing results.

It is easy to derive, from the characterization of the no-free lunch assumption in imperfect models, "free lunch bounds" on the price of contingent claims. For instance, in the model adopted in section 4.3 with transaction costs and a numéraire subject to constraints, we find that if the market consisting of the primitive securities and the contingent claim contains no free lunch, then the ask price for a contingent claim $b$ at date $T$ must be greater than the infimum of the expected values of $\frac{g_{T}}{g_{0}} b$ over all admissible discount processes $g$ in $\mathcal{G}_{J_{\text {Costs }}^{\text {lb }}}$. In an analogous way, we find that the bid price for $b$ must be smaller than the supremum of the expected values of $\frac{g_{T}}{g_{0}} b$ over all admissible discount processes. If the contingent claim $b$ is not subject to any transaction cost then its fair price must lie in the interval $\left[\inf _{g \in \mathcal{G}_{J_{\mathrm{Costs}}^{\mathrm{lb}}}} E\left[\frac{g_{T}}{g_{0}} b\right], \sup _{g \in \mathcal{G}_{J_{\mathrm{Costs}}^{\mathrm{b}}}} E\left[\frac{g_{T}}{g_{0}} b\right]\right]$. The same approach remains valid for the other market imperfections that we have considered. 


\section{Appendix}

Proof of Lemma 2.1 1) We prove it for $\gamma=\gamma_{t} \delta_{t}$ in $\Gamma$. If $\gamma_{t}$ is a simple real random variable, i.e., if it only takes a finite number of values $\left(a_{i}\right)_{i=1}^{I}$, then we can write $\gamma$ in the form $\gamma=\sum_{i=1}^{I} 1_{A_{i}}\left(a_{i} \delta_{t}\right)$ for a partition $\left(A_{i}\right)_{1 \leq i \leq I}$ of $\Omega$, and $\gamma \in S t\left(\Omega, \mathcal{M}_{b}\right)$. For a general $\gamma_{t}, \gamma_{t}$ can be written as the $P$ a.s. limit of a sequence $\left(\gamma_{t}^{n}\right)_{n>0}$ of simple real random variables; then $\gamma=\lim _{n}\left(\gamma_{t}^{n} \delta_{t}\right)$ a.s. $P$, and $\gamma \in M_{P}\left(\Omega, \mathcal{M}_{b}\right)$. Now, since $\|\gamma(\omega)\|_{\mathcal{M}_{b}}=\sup _{\|f\|<1, f \in \mathcal{C}_{0}}\left|\gamma_{t}(\omega) f(t)\right|$, we get $E\left[\|\gamma\|_{\mathcal{M}_{b}}\right]=E\left[\left|\gamma_{t}(\omega)\right|\right]<\infty$.

2) For all $\gamma$ in $\Gamma, \gamma=\sum_{i=1}^{m} \gamma_{t_{i}} \delta_{t_{i}}$, we have $\|\gamma\|_{\mathcal{M}_{b}}(\omega)=\sum_{i=1}^{m}\left|\gamma_{t_{i}}(\omega)\right|$ because for all $\left(t_{1}, \ldots t_{m}\right)$ in $\left(R_{+}\right)^{m}$, we can find $f$ in $\mathcal{C}_{0}$, satisfying $\|f\| \leq 1$ and $f\left(t_{i}\right)=1$ (resp. $\left.f\left(t_{i}\right)=-1\right)$ if $\gamma_{t_{i}}(\omega) \geq 0$ (resp. $\left.\gamma_{t_{i}}(\omega) \leq 0\right)$.

Proof of Theorem 2.5 Let $C \equiv\left(J-\Gamma_{+}\right)$. Assume first that there exists a process $g$ satisfying the conditions of the theorem. Let $x \in \Gamma, x=\lim _{L_{P}^{1}\left(\Omega, \mathcal{M}_{b}\right)} x^{n}, x^{n} \in C$. Since $x^{n} \in C \subseteq \Gamma, E\left[\left|\sum_{t \geq 0} g_{t} x_{t}^{n}-\sum_{t \geq 0} g_{t} x_{t}\right|\right] \leq M\left\|x-x_{n}\right\|_{L_{P}^{1}\left(\Omega, \mathcal{M}_{b}\right)}$ (Lemma $2.1)$, thus $E\left[\sum_{t \geq 0} g_{t} x_{t}^{n}\right] \rightarrow_{n} E\left[\sum_{t \geq 0} g_{t} x_{t}\right]$. Since for all $n, E\left[\sum_{t \geq 0} g_{t} x_{t}^{n}\right] \leq 0$, we get $E\left[\sum_{t \geq 0} g_{t} x_{t}\right] \leq 0$ and $x \notin \Gamma_{+} \backslash\{0\}$.

Suppose now that $\bar{C} \cap \Gamma_{+}=\{0\}$. Let $\mu$ denote any nonnull element of $\Gamma_{+}$. We apply the Hahn-Banach separation theorem in the normed vector space $L_{P}^{1}\left(\Omega, \mathcal{M}_{b}\right)$ to find $\mathfrak{g} \neq 0$ in $\mathcal{L}_{*}^{\infty}\left(\Omega,\left(\mathcal{M}_{b}\right)^{\prime}\right)$, i.e. satisfying

1. $\langle\mathfrak{g}, \nu\rangle_{\left(\mathcal{M}_{b}\right)^{\prime}, \mathcal{M}_{b}}: \omega \rightarrow\langle\mathfrak{g}(\omega), \nu\rangle_{\left(\mathcal{M}_{b}\right)^{\prime}, \mathcal{M}_{b}}$ is measurable for all $\nu$ in $\mathcal{M}_{b}$

2. $\|\mathfrak{g}\|_{\mathcal{L}_{*}^{\infty}\left(\Omega,\left(\mathcal{M}_{b}\right)^{\prime}\right)} \equiv \inf \left\{M \geq 0,\|\mathfrak{g}\|_{\left(\mathcal{M}_{b}\right)^{\prime}} \leq M \quad P\right.$ a.s. $\}<\infty$,

for which, for all $c$ in $C, E\left[\langle\mathfrak{g}, c\rangle_{\left(\mathcal{M}_{b}\right)^{\prime}, \mathcal{M}_{b}}\right] \leq \alpha_{\mu}<\beta_{\mu} \leq E\left[\langle\mathfrak{g}, \mu\rangle_{\left(\mathcal{M}_{b}\right)^{\prime}, \mathcal{M}_{b}}\right]$, where $\langle\mathfrak{g}, c\rangle_{\left(\mathcal{M}_{b}\right)^{\prime}, \mathcal{M}_{b}}: \omega \mapsto\left\langle\mathfrak{g}(\omega)^{\prime}, c(\omega)\right\rangle_{\left(\mathcal{M}_{b}\right)^{\prime}, \mathcal{M}_{b}}$. Since $C$ is a convex cone, we can take $\alpha_{\mu}=0$

Let now for all $t \geq 0, g_{t} \equiv\left\langle\mathfrak{g}, \delta_{t}\right\rangle_{\left(\mathcal{M}_{b}\right)^{\prime}, \mathcal{M}_{b}}$ and $g \equiv\left(g_{t}\right)_{t>0}$. Then, using 1), $g_{t}$ is a measurable random variable. Moreover, using 2), since for all $t \geq 0, \delta_{t} \in \mathcal{M}_{b}$ and satisfies $\left\|\delta_{t}\right\|_{\mathcal{M}_{b}} \leq 1$, we get the existence of $M$ in $R_{+}^{*}$ such that for all $t \geq 0$, $g_{t} \leq M \quad P$ a.s.

We claim that for all $t \geq 0, g_{t}$ is almost surely nonnegative. Suppose that there exists $t \geq 0$ such that $V_{t} \equiv\left\{\left\langle\mathfrak{g}, \delta_{t}\right\rangle_{\left(\mathcal{M}_{b}\right)^{\prime}, \mathcal{M}_{b}}<0\right\}$ has positive $P$-probability. The random variable $\phi_{t}$ in $L_{P}^{1}\left(\Omega, \mathcal{M}_{b}\right)$ given by $\phi_{t}: \omega \rightarrow-1_{V_{t}}(\omega) \delta_{t}$ belongs to $\Gamma_{-}$, so according to the separation theorem $E\left[\left\langle\mathfrak{g}, \phi_{t}\right\rangle_{\left(\mathcal{M}_{b}\right)^{\prime}, \mathcal{M}_{b}}\right] \leq 0$. But $E\left[\left\langle\mathfrak{g}, \phi_{t}\right\rangle_{\left(\mathcal{M}_{b}\right)^{\prime}, \mathcal{M}_{b}}\right]=$ $E\left[-1_{V_{t}}\left\langle\mathfrak{g}, \delta_{t}\right\rangle_{\left(\mathcal{M}_{b}\right)^{\prime}, \mathcal{M}_{b}}\right]>0$, a contradiction.

We claim that for all $\Phi \equiv \sum_{t \geq 0} \Phi_{t} \delta_{t}$ in $J, E\left[\sum_{t \geq 0} g_{t} \Phi_{t}\right] \leq 0$. This is immediate using 2) and the equality $\left\langle\mathfrak{g}(\omega), \sum_{t \geq 0} \Phi_{t} \delta_{t}\right\rangle_{\left(\mathcal{M}_{b}\right)^{\prime}, \mathcal{M}_{b}}=\sum_{t \geq 0} \Phi_{t}(\omega)\left\langle\mathfrak{g}(\omega), \delta_{t}\right\rangle_{\left(\mathcal{M}_{b}\right)^{\prime}, \mathcal{M}_{b}}$.

Denote by $G$ the set of all equivalence classes of processes $g=\left(g_{t}\right)_{t>0}$ such that for all $t, 0 \leq g_{t} \leq M^{g}$ a.s. $P$ and $E\left[\sum_{t \geq 0} g_{t} \Phi_{t}\right] \leq 0$ for all $\Phi \in J$. We claim that for all $s \geq 0$, there exists a process $g^{s}$ in $G$ satisfying $g_{s}^{s}>0$. Fix $s$ in $R_{+}$and let $\mathcal{S}$ be the 
family of equivalence classes of subsets of $\Omega$ formed by the supports of the $g_{s}$ for all $g$ in $G$. We see that the family $\mathcal{S}$ is not reduced to the empty set by considering $\mu=\delta_{s}$ in the separation theorem. The family $\mathcal{S}$ is closed under countable unions: indeed, consider a sequence $\left(g^{n}\right)_{n>0}$ in $G$, and let $\left(a_{n}\right)_{n>0}$ denote a sequence of positive scalars such that $\sum_{n>0} a_{n} M^{g^{n}}<\infty$; the process $h$ given by $h_{t} \equiv \sum_{n>0} a_{n} g_{t}^{n}$ a.s. $P$ for all $t \geq 0$, belongs to $G$. Hence there is $g^{s}$ in $G$ such that for $S^{*}=\left\{g_{s}^{s}>0\right\}$ we have $P\left(S^{*}\right)=\sup \{P(S) ; S \in \mathcal{S}\}$. We shall now prove that $P\left(S^{*}\right)=1$. If $P\left(S^{*}\right)<1$, then we can apply the separation theorem to $\mu=1_{\left(\Omega-S^{*}\right)} \delta_{t}$ which belongs to $\Gamma_{+} \backslash\{0\}$ and proceeding as above, we find $g^{\prime s} \in G$ such that $E\left[1_{\left(\Omega-S^{*}\right)} g_{s}^{\prime s}\right]>0$. Then, $g^{s}+g^{s}$ would be an element of $G$, whose support has $P$-probability strictly greater than $P\left(S^{*}\right)$ : a contradiction.

In the same way, we get that there exists $g$ in $G$ such that $g_{d_{n}}>0$ almost surely for all $d_{n} \in d$, where $d$ is the sequence introduced in Assumption A. We consider the process $g$ such that for all $t \geq 0, g_{t} \equiv \sum_{n>0} b_{n} g_{t}^{d_{n}}$, where $\left(b_{n}\right)_{n>0}$ is a sequence of positive scalars such that $\sum_{n \geq 0} b_{n} M^{g_{d_{n}}}<\infty$.

We have found so far a process $g$ such that for all $t \geq 0, g_{t}$ is in $L_{+}^{\infty}(\Omega, F, P ; R)$, $g_{t} \leq M$ for all $t, g_{d_{n}}>0$ almost surely for all $d_{n} \in d$ and $E\left[\sum_{t \geq 0} g_{t} \Phi_{t}\right] \leq 0$ for all $\Phi=\sum_{t \geq 0} \Phi_{t} \delta_{t}$ in $J$. Then it is easy to check that the same holds for the $\left(F_{t}\right)_{t \geq 0^{-}}$ adapted process $\tilde{g}=\left(\tilde{g}_{t}\right)_{t>0}$ defined by $\tilde{g}_{t}=E\left[g_{t} \mid F_{t}\right] \quad P$ a.s. for all $t$. To finish the proof, we only need to show that for all $t \geq 0, \tilde{g}_{t}>0 \quad P$ a.s.. Assume that for some $T$ outside the set of dates $\left\{d_{n} ; n \in N\right\}$ we have just considered, the event $B_{T} \equiv\left\{\tilde{g}_{T}=0\right\}$ has positive $P$-probability; we know that there exists $\Phi=\sum_{t>0} \Phi_{t} \delta_{t}$ in $C$ such that $\Phi_{T}=0$ outside $B_{T}, \Phi_{t}=0$ for all $t<T, \Phi_{t} \geq 0$ for all $t>T, \exists \bar{d}_{n} \in d$, $P\left[\Phi_{d_{n}}>0\right]>0$. For this particular $\Phi$, we would have $E\left[\sum_{t \geq 0} g_{t} \Phi_{t}\right] \geq E\left[g_{d_{n}} \Phi_{d_{n}}\right]>$ 0 , which is impossible and completes our proof. $\square$

Proof of Corollary 2.6 The proof remains the same as for Theorem 2.5, replacing the sequence $\left(d_{n}\right)_{n \geq 0}$ by $N$.

Proof of Lemma 3.1 The set $\mathcal{G}_{J^{\mathrm{nu}} \mathrm{m}}$ consists of all adapted process $g \in \mathcal{G}$ such that for all $\Phi$ in $J^{\mathrm{num}}, E\left[\sum_{t \geq 0} \Phi_{t} g_{t}\right] \leq 0$. Applying this inequality to $\Phi^{\left(0 ; 1_{A}, t_{1}, t_{2}\right)}$ and $\Phi^{\left(0 ;-1_{A}, t_{1}, t_{2}\right)}$ for all $t_{1} \leq t_{2}$, and $A \in F_{t_{1}}$, we get that for all $t_{1} \leq t_{2}, E\left[1_{A} S_{t_{2}}^{0} g_{t_{2}}\right] \leq$ $E\left[1_{A} g_{t_{1}} S_{t_{1}}^{0}\right]$ and $E\left[1_{A} S_{t_{2}}^{0} g_{t_{2}}\right] \geq E\left[1_{A} g_{t_{1}} S_{t_{1}}^{0}\right]$, thus the process $\left(g_{t} S_{t}^{0}\right)_{t \geq 0}$ is a $P$ martingale for $\left(F_{t}\right)_{t \geq 0}$. The converse is immediate.

Proof of Corollary 3.2 For 1) and 2), one implication is immediate taking

$g_{t} \equiv 1 / S_{t}^{0} E\left[d \bar{P} / d P \mid F_{t}\right]$ and applying Lemma 3.1 and Theorem 2.5. For the converse implication, we know by Lemma 3.1 that for all $g \in \mathcal{G}_{J^{\mathrm{num}}}, g S^{0}$ is a martingale. The process $g S^{0}$ admits a right-continuous modification, that can be chosen so as to be a martingale with respect to $\left(F_{t}\right)_{t \geq 0}$ (see Karatzas-Shreve (1991)). For 1), the process $\left(g_{t} S_{t}^{0}\right)_{t \geq 0}$ is uniformly integrable, so it converges $P$ a.s. to an integrable random variable that we shall denote by $g_{\infty} S_{\infty}^{0}$, such that $\left\{g_{t} S_{t}^{0} ; F_{t} ; 0 \leq t \leq \infty\right\}$ is a martingale (see Karatzas-Shreve (1991)). Since for all $t \geq 0, g_{t} S_{t}^{0}$ is $P$ a.s. positive, we define an absolutely continuous probability measure $\bar{P}$ on $(\Omega, F)$ by setting $\frac{d \bar{P}}{d P} \equiv$ $\frac{g_{\infty} S_{\infty}^{0}}{E\left[g_{\infty} S_{\infty}^{0}\right]}$. Its density restricted to any $F_{t}$, which is equal to $\frac{g_{t} S_{t}^{0}}{E\left[g_{\infty} S_{\infty}^{0}\right]}$, is positive. Then for all investment $\Phi$ in $J, \frac{\Phi_{t}}{S_{t}^{0}}$ belongs to $L^{1}\left(\Omega, F_{t}, \bar{P}\right)$ for all $t \geq 0$ and $E^{\bar{P}}\left[\sum_{t \geq 0} \frac{\Phi_{t}}{S_{t}^{0}}\right]=$ $\frac{1}{E\left[g_{\infty} S_{\infty}^{0}\right]} E\left[\sum_{t \geq 0} \Phi_{t} g_{t}\right] \leq 0$. 
For 2),

Proof of Lemma 3.3 Any $g \in \mathcal{G}_{J^{\mathrm{b}}}$ satisfies $E\left[\sum_{t \geq 0} g_{t} \Phi_{t}^{\left(0 ; 1_{A}, t_{1}, t_{2}\right)}\right] \leq 0$ for all $t_{1} \leq t_{2}$, for all $A \in F_{t_{1}}$. Then for all $A \in F_{t_{1}}, E\left[1_{A} g_{t_{2}} S_{t_{2}}^{0}\right] \leq E\left[1_{A} g_{t_{1}} S_{t_{1}}^{0}\right]$ and the process $g S^{0}$ is a supermartingale. In an analogous way, we find that the process $g S^{1}$ is a submartingale.

Proof of Lemma 3.4 Since $(C 3)$ implies $(C 1)$, using lemma 3.51$)$, there is a right-continuous process $g \in \mathcal{G}$ such that for all $t_{1} \leq t_{2}, E\left[g_{t_{2}}\left(\frac{S_{t_{2}}^{0}}{S_{t_{1}}^{0}}-\frac{S_{t_{2}}^{1}}{S_{t_{1}}^{1}}\right) \mid F_{t_{1}}\right] \leq$ 0. Then,

$$
\lim _{t_{2} \rightarrow t_{1}} E\left[g_{t_{2}} \frac{\left(\frac{S_{t_{2}}^{0}}{S_{t_{1}}^{0}}-\frac{S_{t_{2}}^{1}}{S_{t_{1}}^{1}}\right)}{t_{2}-t_{1}} \mid F_{t_{1}}\right]=E\left[g_{t_{1}}\left(r_{t_{1}}^{0}-r_{t_{1}}^{1}\right) \mid F_{t_{1}}\right]
$$

$=g_{t_{1}}\left(r_{t_{1}}^{0}-r_{t_{1}}^{1}\right) \leq 0$, so that for all $s \geq 0, r_{s}^{0} \leq r_{s}^{1}$ a.s. $P$. Now, let $A$ be given by $A \equiv\left\{(s, \omega), r_{s}^{0}(\omega)>r_{s}^{1}(\omega)\right\}$. Since $r^{0}$ and $r^{1}$ are both progressively measurable, we know that $A \in \mathcal{B}([0, t]) \otimes F_{t}$ for all $t \geq 0$. By Fubini's theorem, letting $\lambda$ denote the Lebesgue measure, we get that $\lambda \otimes P(A)=0$, so that by Fubini's Theorem again, $\lambda\left(A_{\omega}\right)=0$ a.s. $P$ (where as usual, $A_{\omega} \equiv\{s,(s, \omega) \in A\}$ ) and for all $t_{1} \leq t_{2}$, $\int_{t_{1}}^{t_{2}} r_{s}^{0} d s \leq \int_{t_{1}}^{t_{2}} r_{s}^{1} d s P$ a.s. $\square$

Proof of Lemma 3.5 1$)$ Let $s \geq 0$ be fixed. Using Condition $(C 1)$ and proceding like in Lemma 3.3, we get that $X_{t} \equiv\left(g_{t} \frac{S_{t}^{0}}{S_{s}^{0}}\right)_{t \geq s}$ is an $\left(F_{t}\right)_{t \geq s}$-supermartingale and $Y_{t} \equiv\left(g_{t} \frac{S_{t}^{1}}{S_{s}^{1}}\right)_{t \geq s}$ is an $\left(F_{t}\right)_{t \geq s}$-submartingale. We know that $P$ a.s., the limits $X_{s+}$ and $Y_{s+}$ exist. By considering the investments $\Phi{ }^{\left(k ; \frac{1}{S_{s}^{k}}, s, t\right)} \in J^{\mathrm{lb}}$, we get for all $t \geq s$, $E\left[X_{t} \mid F_{s}\right] \leq g_{s} \leq E\left[Y_{t} \mid F_{s}\right]$. Let $\left\{s_{n}\right\}$ be a sequence of rational numbers decreasing to $s$. Then $E\left[Y_{s_{n}}\right]$ is decreasing and bounded below by $E\left[Y_{s}\right]$. Then $\left\{Y_{s_{n}}\right\}$ is a u.i. sequence and a.s. convergence implies $L^{1}$ convergence. Proceding in the same way for $X$, we get

$$
E\left[\frac{\left(g S^{0}\right)\left(s_{+}\right)}{S_{s}^{0}} \mid F_{s}\right] \leq g_{s} \leq E\left[\frac{\left(g S^{1}\right)\left(s_{+}\right)}{S_{s_{+}}^{1}} \mid F_{s}\right]
$$

and, using the right-continuity of the filtration, $g_{s_{+}}$exists and $g_{s}=g_{s_{+}}$a.s. $P$. thus $g$ admits a right-continuous modification.

2) We prove it for $s=0$. We know by Lemma 3.3 and 1) that for $g \in \mathcal{G}_{J^{\mathrm{b}}}$, $g S^{0}$ (resp. $g S^{1}$ ) is a right-continuous super-(resp. sub-)martingale . Moreover, by Condition $(C 2)$, we have $g S^{0} \leq g S^{1}$. Then we use the approach adopted in JouiniKallal (1995a, Lemma 3) or Choulli-Stricker (1997), adapting it to the case of infinite time to get the existence of a right-continuous martingale process $\left(m_{t}\right)_{t \geq 0}$ which satisfies $\left(g S^{0}\right)(t) \leq m_{t} \leq\left(g S^{1}\right)(t)$ for all $t \geq 0$ (see Lemma 1 in Appendix B for a detailed proof). Since for all $t \geq 0, g_{t}$ is almost surely positive, we may define the process $\left(Z_{t}\right)_{t \geq 0}$ by $Z_{t} \equiv \frac{m_{t}}{g_{t}}$ for all $t \geq 0$.

3) Consider a set of dates $\left(t_{i}\right)_{i<N}$ in $[0, T]$ such that $0=t_{0}<t_{1}<\ldots<t_{N}=T$, where $T$ is a given positive real number. For all $i \geq 0$, we know that there exists a process denoted by $Y^{i}$ defined for $t \geq t_{i}$, which is a $P$-martingale for $\left(F_{t}\right)_{t \geq t_{i}}$ and such 
that $g_{t} \frac{S_{t}^{0}}{S_{t_{i}}^{0}} \leq Y_{t}^{i} \leq g_{t} \frac{S_{t}^{1}}{S_{t_{i}}^{1}} \quad$ for all $t \geq t_{i}$. Now let $\tilde{Y}$ denote the $\left(F_{t}\right)_{t \in\left(t_{i}\right)_{i \leq N}}$-adapted process defined for $t \in\left(t_{i}\right)_{i \leq N}$ by $\tilde{Y}_{t}=\prod_{j \leq i} \frac{Y_{t_{j}}^{j-1}}{g_{t_{j}}} Y_{t}^{i}$ for all $t_{i} \leq t<t_{i+1}$. It is easy to check that $\tilde{Y}$ is a $P$-martingale for $\left(F_{t}\right)_{t \in\left(t_{i}\right)_{i \leq N}}$. Moreover, if we let $X^{0} \triangleq \frac{\tilde{Y}}{g}$, then for all $i \leq N-1, \frac{X_{t_{i+1}}^{0}}{X_{t_{i}}^{0}}=\frac{Y_{t_{i+1}}^{i}}{g_{t_{i+1}}}$, so that for all $i \leq N-1$,

$$
\frac{S_{t_{i+1}}^{0}}{S_{t_{i}}^{0}} \leq \frac{X_{t_{i+1}}^{0}}{X_{t_{i}}^{0}} \leq \frac{S_{t_{i+1}}^{\prime 0}}{S_{t_{i}}^{\prime 0}}
$$

We extend the process found this way for each time grid $t_{0}<t_{1}<\ldots<t_{N}$ into a continuous time process, defined for $t \leq T$, by letting $X^{0}=\exp \int_{0}^{*} y_{s} d s$ with $y(s)=$ $y_{i}(s)$ for every $t_{i} \leq t<t_{i+1}$ where $\frac{X_{t_{i+1}}^{0}}{X_{t_{i}}^{0}}=\exp \int_{t_{i}}^{t_{i+1}} y_{i}(s) d s$. Note that $X^{0}$ is absolutely continuous and can be written $X_{t}^{0}=1+\int_{0}^{t} u_{s} d s$ where $u_{s}=y_{s} \exp \int_{0}^{s} y_{v} d v$. We do this for finer and finer grids of union equal to some dense subset of $[0, T]$. The sequence of slopes $u(s, \omega)$ constructed this way is bounded in $L^{\infty}([0, T] \times \Omega)$, so it admits a convergent (for the weak-star topology $\sigma\left(L^{\infty}, L^{1}\right)$ ) subsequence. Let us denote by $\tilde{u}$ its limit, and consider the process $Z=1+\int_{0}^{*} \tilde{u}_{s} d s$, defined for $t \leq T$, which can be written in the form $Z_{t}=\exp \int_{0}^{t} r_{s} d s$, for some bounded process $r$. The "associated" subsequence $g X^{0}$ is also convergent for the weak-star topology $\sigma\left(L^{\infty}, L^{1}\right)$ with limit equal to $g Z$. Using the fact that for all time grid $t_{0}<t_{1}<$ $\ldots<t_{N}$, the associated process $g X^{0}$ is a $P$-martingale for $\left(F_{t}\right)_{t \in\left\{t_{0}, \ldots, t_{N}\right\}}$, it is easy to see that $g Z$ is a $P$-martingale for $\left(F_{t}\right)_{t<T}$. We adopt the same approach between $n T$ and $(n+1) T$ for $n \geq 1$, to find a process $Z$, defined for $t \geq 0$, which can be written in the form $Z_{t}=\exp \int_{0}^{t} r_{s} d s$, for some bounded process $r$ and such that $g Z$ is a $P$-martingale for $\left(F_{t}\right)_{t \geq 0}$. Proceding like in the proof of Lemma 3.4, we get that $r_{s}^{0} \leq r_{s} \leq r_{s}^{1}$.

\section{Proof of Lemma 3.6 Immediate.}

Proof of Theorem 4.2 Using Theorem 2.5, for all $A \in F_{t_{1}}$ and $x \in \mathcal{C}$, we have $E\left[1_{A} x \cdot\left(g_{t_{2}} S_{t_{2}}-g_{t_{1}} S_{t_{1}}\right)\right] \leq 0$ so that for all $x \in \mathcal{C}, x \cdot E\left[\left(g_{t_{2}} S_{t_{2}}-g_{t_{1}} S_{t_{1}}\right) \mid F_{t_{1}}\right] \leq$ 0 and $E\left[g_{t_{2}} S_{t_{2}}-g_{t_{1}} S_{t_{1}} \mid F_{t_{1}}\right] \in \mathcal{C}^{0}$. Conversely, if $E\left[g_{t_{2}} S_{t_{2}}-g_{t_{1}} S_{t_{1}} \mid F_{t_{1}}\right] \in \mathcal{C}^{0}$, then for all $A \in F_{t_{1}}$ and $x \in \mathcal{C}, 1_{A} x \cdot E\left[g_{t_{2}} S_{t_{2}}-g_{t_{1}} S_{t_{1}} \mid F_{t_{1}}\right] \leq 0$ a.s. $P$, thus $E\left[\sum_{t \geq 0}\left(x \cdot \Phi^{\left(A, t_{1}, t_{2}\right)}\right)_{t} g_{t}\right] \leq 0$ and Theorem 2.5 concludes.

Proof of Corollary 4.3 Immediate using Theorem 4.2.

Proof of Theorem 4.4 One implication is easy noticing that for all stopping times $\tau_{1}$ and $\tau_{2}$, we have, using the fact that $\tilde{S}$ lies between the bid and ask price processes, the law of iterated expectations and the optional sampling theorem,

$$
E\left[\sum_{t \geq 0} \Phi_{t}^{\left(k ; \tau_{1}, \tau_{2}\right)} g_{t}\right] \leq E\left[S_{\tau_{2}}^{\prime k} g_{\tau_{2}}-S_{\tau_{1}}^{k} g_{\tau_{1}}\right] \leq E\left[\tilde{S}_{\tau_{2}}^{k} g_{\tau_{2}}-\tilde{S}_{\tau_{1}}^{k} g_{\tau_{1}}\right] \leq 0 .
$$

The result then follows from Theorem 2.5.

For the converse implication, there exists a process $g=\left(g_{t}\right)_{t \geq 0} \in \mathcal{G}$ such that for all $k$, for all $\tau_{1}$ and $\tau_{2}$ in $S_{t, \infty}^{f}, E\left[-S_{\tau_{1}}^{k} g_{\tau_{1}}+S_{\tau_{2}}^{\prime k} g_{\tau_{2}} \mid F_{t}\right] \leq 0$ or

$$
E\left[S_{\tau_{2}}^{\prime k} g_{\tau_{2}} \mid F_{t}\right] \leq E\left[S_{\tau_{1}}^{k} g_{\tau_{1}} \mid F_{t}\right]
$$


We consider the two $n$-dimensional processes $Z$ and $Z^{\prime}$ given by $Z_{t}^{\prime}=e s s \sup E\left[S_{\tau}^{\prime} g_{\tau} \mid F_{t}\right]$ $\tau \in S_{t, \infty}^{f}$ and $Z_{t}=\underset{\tau \in S_{t, \infty}^{f}}{e s s i n f} E\left[S_{\tau} g_{\tau} \mid F_{t}\right]$ for all $t \geq 0$. In words, $Z^{\prime k}$ is the supremum of the con-

ditional expected value of the proceeds from the strategy that consists in going short in one security $k$ (and investing the proceeds in security 0 ) after time $t$ (but not necessarily at the same time in different events). The process $Z$ is defined symmetrically. We have for all $t \geq 0, S_{t}^{\prime} g_{t} \leq Z_{t}^{\prime}$ as well as $Z_{t} \leq S_{t} g_{t}$ because for all $t \geq 0$, the stopping time $\tau=t$ belongs to $S_{t, \infty}^{f}$.

It is a standard result in optimal stopping that $Z^{\prime}$ is a $P$-supermartingale for $\left(F_{t}\right)_{t>0}$ and that $Z$ is a $P$-submartingale for $\left(F_{t}\right)_{t>0}$. Using inequality (5.1), we have $Z^{\prime} \leq Z$. The process $g$ can be taken right-continuous (Lemma 3.5, 1.) Adapting Lemma 3 in Jouini-Kallal (1995a), we get that there is a process $\tilde{Z}$ lying between $\left(S^{\prime} g\right)$ and $(S g)$, which is a $P$-martingale for $\left(F_{t}\right)_{t \geq 0}$ (Lemma 1 in Appendix B). Now, letting $\tilde{S} \equiv \frac{\tilde{Z}}{g}$, we get that $g \tilde{S}$ is a right-continuous $P$-martingale and for all $t \geq 0, S_{t}^{\prime} \leq \tilde{S}_{t} \leq S_{t} P$ a.s. $\square$

\section{Appendix B}

Lemma 1 If $Z$ (resp. $Z^{\prime}$ ) is an $\left\{\left(F_{t}\right)_{t \geq 0}, P\right\}$-supermartingale (resp. submartingale) such that $Z_{t}^{\prime} \leq Z_{t}$ for all $t \geq 0$, then there exists an $\left\{\left(F_{t}\right)_{t \geq 0}, P\right\}$-martingale process $\tilde{Z}$ satisfying $Z_{t+}^{\prime} \leq \tilde{Z}_{t} \leq Z_{t+}$ for all $t \geq 0$.

Proof We adapt here the approach of Jouini-Kallal (1995a) to the case of infinite and possibly not right continuous processes. If the processes are discrete then the result is an application of the intermediate value Theorem. Otherwise, consider an increasing sequence of time grids $D_{n}=\left\{\frac{k}{2^{n}} ; k \geq 0\right\}$ which converges to a dense subset $D$ of $R_{+}$. Then proceed like in Jouini-Kallal (1995a) to find a process $Y$ defined on $D$, which is a $P$-martingale for $\left(F_{t}\right)_{t \in D}$ and which satisfies $Z_{t}^{\prime} \leq Y_{t} \leq Z_{t}$ for all $t \in D$. Then extend this process to $R_{+}$by defining for all $t \notin D, \tilde{Z}_{t}=\lim _{\substack{s \rightarrow t, s>t \\ s \in D}} Y_{s}$; we recall that for any submartingale $X=\left\{X_{t}, F_{t}, t \geq 0\right\}, P$ almost surely the limit $X_{t+} \triangleq \lim _{\substack{s \rightarrow t, s>t \\ s \in Q}}$ exists for all $t \geq 0$. The process $\tilde{Z}$ lies between $Z_{+}^{\prime}$ and $Z_{+}$. Using the right-continuity of the filtration and the fact that $Y$ itself is a martingale, we obtain that $\tilde{Z}$ is a martingale.

\section{References}

[1] Adler, I., Gale, D. (1997): Arbitrage and growth rate for riskless investments in a stationary economy. Math. Fin. 2, 73-81.

[2] Ansel, J.P, Stricker, C (1990): Quelques remarques sur un théorème de Yan. Sém. de probabilités. Lecture notes in Mathematics XXIV Springer-Verlag, New-York, 226-274.

[3] Brannath, W. (1997): No arbitrage and martingale measures in option pricing. Dissertation zur Erlangung des akademischen Grades. Universität Wien.

[4] Cantor, D.G., Lippman, S.A. (1983): Investment selection with imperfect capital markets. Econometrica, 51, 1121-1144. 
[5] Cantor, D.G., Lippman, S.A. (1995): Optimal investment selection with a multitude of projects. Econometrica, 63-5, 1231-1241.

[6] Carassus, L., Jouini, E. (1997): Coûts de transaction et contraintes de vente à découvert : une approche unifiée. DP CREST n ${ }^{\circ} 9758$.

[7] Choulli, T., Stricker, C. (1997): Séparation d'une sur- et d'une sousmartingale par une martingale. Thèse de T. Choulli. Université de Franche-Comté.

[8] Cvitanic, J., Karatzas, I. (1993): Hedging contingent claims with constrained portfolios. Ann. Appl. Prob., 3, 652-681.

[9] Cvitanic, J., Karatzas, I. (1996): Hedging and portfolio optimization under transaction costs: a martingale approach. Math. Fin. 6, 133-166.

[10] Dalang, R.C., Morton, A., Willinger, W. (1989): Equivalent martingale measures and no arbitrage in stochastic securities market models. Stochastics and Stochastic Rep. 29, 185-202.

[11] Delbaen, F. (1992): Representing martingale measures when asset prices are continuous and bounded. Math. Fin. 2, 107-130.

[12] Delbaen, F., Kabanov, Y., Valkeila, E. (1998): Hedging under transaction costs in currency markets : a discrete-time model. Preprint.

[13] Delbaen, F., Schachermayer, W. (1994): A general version of the fundamental theorem of asset pricing. Math. Ann. 300, 463-520.

[14] Delbaen, F., Schachermayer, W. (1998): The fundamental theorem of asset pricing for unbounded stochastic processes. Math. Ann. 312, 215 - 250.

[15] Dermody, J.-C., Rockafellar, R.T. (1991): Cash stream valuation in the face of transaction costs and taxes. Math. Fin. 1, 31-54.

[16] Dermody, J.-C., Rockafellar, R.T. (1995): Tax basis and nonlinearity in cash stream. Math. Fin. 5, 97-119.

[17] Diestel, J., Uhl, J.J. (1977): Vector measures. Math. Surveys, Amer. Math. Soc.

[18] Duffie, D., Huang, C. (1986): Multiperiod security markets with differential information: martingales and resolution times. J. Math. Econ., 15, 283-303.

[19] Föllmer, H., Kramkov, K. (1997): Optional decomposition under constraints. Probab. Theory Relat. Fields 109, 1-25.

[20] Frittelli, M., Lakner, P. (1994): Arbitrage and free lunch in a general financial market model; the fundamental theorem of asset pricing. In Mathematical Finance (M.H.A. Davis, D. Duffie, W.H. Fleming and S.E. Shreve, Eds. Springer, NY.

[21] Harrison, M., Kreps, D. (1979): Martingales and arbitrage in multiperiod security markets. J. Econ. Theory 20, 381-408.

[22] Harrison, M., Pliska, S. (1981): Martingales and stochastic integrals in the theory of continuous trading. Stochastic Processes Appl. 11, 215-260. 
[23] Jouini, E., Kallal, H. (1995a): Martingales and arbitrage in securities markets with transaction costs. J. Econ. Theory 66, 178-197.

[24] Jouini, E., Kallal, H. (1995b): Arbitrage in securities markets with short-sales constraints. Math. Fin. 5, 197-232.

[25] Kabanov, Y. (1998): Hedging and liquidation under transaction costs in currency markets. To appear Finance and Stochastics, 3.

[26] Karatzas, I., Shreve, S.E. (1991): Brownian motion and stochastic calculus. Graduate texts in Mathematics. Springer.

[27] Kreps, D. (1981): Arbitrage and equilibrium in economies with infinitely many commodities. J. Math. Econ. 8, 15-35.

[28] Marle, C.M. (1974): Mesures et probabilités, Hermann.

[29] Pham, H., Touzi, N. (1996): The fundamental theorem of asset pricing with cone constraints. Forthcoming in J. Math. Econ.

[30] Schachermayer, W. (1994): Martingale measures for discrete time processes with infinite horizon. Math. Fin. 4, 25-55

[31] Schwartz, L. (1974-1975): Fonctions mesurables et $*$-scalairement mesurables, propriété de Radon-Nikodym, Exposés 4, 5 et 6, Sém. Maurey-Schwartz, Ecole Polytechnique.

[32] Stricker, C. (1990): Arbitrage et lois de martingale. Ann. Inst. Henri Poincaré 26, 451-460.

[33] Yan, J.A. (1980): Caractérisation d'une classe d'ensembles convexes de $L^{1}$ ou $H^{1}$. Sém. de Probabilités. Lecture notes in Mathematics XIV 784, 220-222 